\title{
MSSM Higgs boson decays to bottom quark pairs reexamined
}

\author{
Jaume Guasch \\ Paul Scherrer Institut, CH-5232 Villigen PSI, Switzerland \\ Petra Häfliger \\ Paul Scherrer Institut, CH-5232 Villigen PSI, Switzerland \\ and Institute for Particle Physics, ETH Zürich, CH-8093 Zürich, Switzerland \\ Michael Spira \\ Paul Scherrer Institut, CH-5232 Villigen PSI, Switzerland
}

(Received 12 June 2003; revised manuscript received 19 September 2003; published 11 December 2003)

\begin{abstract}
We present an update of neutral Higgs boson decays into bottom quark pairs in the minimal supersymmetric extension of the standard model. In particular the resummation of potentially large higher-order corrections due to the soft supersymmetry (SUSY) breaking parameters $A_{b}$ and $\mu$ is extended. The remaining theoretical uncertainties due to unknown higher-order SUSY-QCD corrections are analyzed quantitatively.
\end{abstract}

DOI: 10.1103/PhysRevD.68.115001

PACS number(s): 14.80.Cp, 12.60.Jv, 14.80.Ly

\section{INTRODUCTION}

The Higgs mechanism is a cornerstone of the standard model (SM) and its supersymmetric extensions. The search for Higgs bosons is one of the most important endeavors at future high-energy experiments. Since the minimal supersymmetric extension of the standard model (MSSM) requires the introduction of two Higgs doublets in order to preserve supersymmetry (SUSY), there are five elementary Higgs particles: two $C P$-even $(h, H)$, one $C P$-odd $(A)$ and two charged ones $\left(H^{ \pm}\right)$. At lowest order all couplings and masses of the MSSM Higgs sector are fixed by two independent input parameters, which are generally chosen as $\operatorname{tg} \beta$ $=v_{2} / v_{1}$, the ratio of the two vacuum expectation values $v_{1,2}$, and the pseudoscalar Higgs-boson mass $M_{A}$. At leading order (LO) the light scalar Higgs boson mass $M_{h}$ has to be smaller than the $Z$-boson mass $M_{Z}$. Including the oneloop and dominant two-loop corrections the upper bound is increased to $M_{h} \lesssim 135 \mathrm{GeV}$ [1]. The couplings of the various neutral Higgs bosons to fermions and gauge bosons depend on the angles $\alpha$ and $\beta$. Normalized to the SM Higgs boson couplings, they are listed in Table I.

The pseudoscalar particle $A$ does not couple to gauge bosons at tree level, and its couplings to down-type (up-type) fermions are (inversely) proportional to $\operatorname{tg} \beta$. The negative direct searches for the Higgsstrahlung processes $e^{+} e^{-}$ $\rightarrow Z h, Z H$ and the associated production $e^{+} e^{-} \rightarrow A h, A H$ yield lower bounds of $M_{h, H}>91.0 \mathrm{GeV}$ and $M_{A}$ $>91.9 \mathrm{GeV}$. The range $0.5<\operatorname{tg} \beta<2.4$ in the MSSM is excluded by the Higgs boson searches for a SUSY scale $M_{S U S Y}=1 \mathrm{TeV}$ at the CERN $e^{+} e^{-}$collider LEP2 experiments [2].

The scalar superpartners $\widetilde{f}_{L, R}$ of the left- and right-handed fermion components mix with each other. The mass eigen-

\footnotetext{
${ }^{1}$ The excluded range of $\operatorname{tg} \beta$ values depends significantly on the value of the top-quark mass [3].
}

states $\widetilde{f}_{1,2}$ of the sfermions $\widetilde{f}$ are related to the current eigenstates $\widetilde{f}_{L, R}$ by mixing angles $\theta_{f}$,

$$
\begin{aligned}
& \widetilde{f}_{1}=\widetilde{f}_{L} \cos \theta_{f}+\widetilde{f}_{R} \sin \theta_{f} \\
& \widetilde{f}_{2}=-\widetilde{f}_{L} \sin \theta_{f}+\widetilde{f}_{R} \cos \theta_{f},
\end{aligned}
$$

which are proportional to the masses of the ordinary fermions. Thus mixing effects are only important for the thirdgeneration sfermions $\tilde{t}, \tilde{b}, \tilde{\tau}$, the mass matrix of which is given $^{2}$ by [4]

$$
\mathcal{M}_{\tilde{f}}=\left[\begin{array}{cc}
M_{\tilde{f}_{L}}^{2}+m_{f}^{2} & m_{f}\left(A_{f}-\mu r_{f}\right) \\
m_{f}\left(A_{f}-\mu r_{f}\right) & M_{\widetilde{f}_{R}}^{2}+m_{f}^{2}
\end{array}\right],
$$

with the parameters $r_{b}=r_{\tau}=1 / r_{t}=\operatorname{tg} \beta$. The parameters $A_{f}$ denote the trilinear scalar coupling of the soft supersymmetry breaking part of the Lagrangian. Consequently the mixing angles acquire the form

$$
\sin 2 \theta_{f}=\frac{2 m_{f}\left(A_{f}-\mu r_{f}\right)}{M_{\tilde{f}_{1}}^{2}-M_{\tilde{f}_{2}}^{2}}, \quad \cos 2 \theta_{f}=\frac{M_{\tilde{f}_{L}}^{2}-M_{\tilde{f}_{R}}^{2}}{M_{\tilde{f}_{1}}^{2}-M_{\tilde{f}_{2}}^{2}}
$$

and the masses of the squark mass eigenstates are given by

$$
\begin{aligned}
M_{\widetilde{f}_{1,2}}^{2}= & m_{f}^{2}+\frac{1}{2}\left[M_{\widetilde{f}_{L}}^{2}+M_{\widetilde{f}_{R}}^{2}\right. \\
& \left.\mp \sqrt{\left(M_{\widetilde{f}_{L}}^{2}-M_{\tilde{f}_{R}}^{2}\right)^{2}+4 m_{f}^{2}\left(A_{f}-\mu r_{f}\right)^{2}}\right] .
\end{aligned}
$$

The neutral Higgs boson couplings to sfermions read as [5]

\footnotetext{
${ }^{2}$ For simplicity, the $D$ terms have been absorbed in the sfermion mass parameters $M_{\tilde{f}_{L / R}}^{2}$.
} 
TABLE I. Higgs boson couplings in the MSSM to fermions and gauge bosons $[V=W, Z]$ relative to SM couplings.

\begin{tabular}{lcccc}
\hline \hline & & $g_{u}^{\Phi}$ & $g_{d}^{\Phi}$ & $g_{V}^{\Phi}$ \\
\hline SM & $H$ & 1 & 1 & 1 \\
MSSM & $h$ & $\cos \alpha / \sin \beta$ & $-\sin \alpha / \cos \beta$ & $\sin (\beta-\alpha)$ \\
& $H$ & $\sin \alpha / \sin \beta$ & $\cos \alpha / \cos \beta$ & $\cos (\beta-\alpha)$ \\
& $A$ & $1 / \operatorname{tg} \beta$ & $\operatorname{tg} \beta$ & 0 \\
\hline \hline
\end{tabular}

$$
\begin{aligned}
& g_{\tilde{f}_{L} \tilde{f}_{L}}^{\Phi}=m_{f}^{2} g_{1}^{\Phi}+M_{Z}^{2}\left(I_{3 f}-e_{f} \sin ^{2} \theta_{W}\right) g_{2}^{\Phi} \\
& g_{\tilde{f}_{R} \tilde{f}_{R}}^{\Phi}=m_{f}^{2} g_{1}^{\Phi}+M_{Z}^{2} e_{f} \sin ^{2} \theta_{W} g_{2}^{\Phi} \\
& g_{\tilde{f}_{L} \tilde{f}_{R}}^{\Phi}=-\frac{m_{f}}{2}\left(\mu g_{3}^{\Phi}-A_{f} g_{4}^{\Phi}\right),
\end{aligned}
$$

with the couplings $g_{i}^{\Phi}$ listed in Table II.

In this paper we investigate the theoretical status of SUSY-QCD corrections to neutral Higgs boson decays into bottom quark pairs. In particular we concentrate on the theoretical uncertainties of the partial width in regions, where the SUSY-QCD corrections are large, i.e. for large values of $\operatorname{tg} \beta$ and sizeable magnitudes of the Higgsino mass parameter $\mu$ [6]. These regions are particularly interesting, since the contributions generated by gluino exchange are enhanced by $\operatorname{tg} \beta$. They play an important role in the phenomenology of SUSY Higgs bosons at high-energy colliders, since they shift the Higgs-boson discovery and exclusion regions significantly [7]. The corrections can also provide a distinction between supersymmetric and non-supersymmetric Higgs bosons. The dominant contributions have been resummed before [8]. However, the trilinear coupling $A_{b}$ may be large, too. We extend the resummation by including the dominant $A_{b}$ terms.

Although we investigate only the SUSY-QCD corrections, it should be noted that the electroweak corrections can be important, too, and yield an additional contribution to the uncertainties. The full one-loop electroweak corrections were computed in Ref. [9], and later refined in [10] including the two-loop contributions to the Higgs boson propagator matrix. Section II summarizes the present theoretical status of Higgs boson decays into bottom quark pairs and sets the basis for the resummation, which is described in Sec. III. In Sec. IV we analyze the remaining theoretical uncertainties

TABLE II. Coefficients of the neutral MSSM Higgs boson couplings to sfermion pairs.

\begin{tabular}{cccccc}
\hline \hline$\tilde{f}$ & $\Phi$ & $g_{1}^{\Phi}$ & $g_{2}^{\Phi}$ & $g_{3}^{\Phi}$ & $g_{4}^{\Phi}$ \\
\hline & $h$ & $\cos \alpha / \sin \beta$ & $-\sin (\alpha+\beta)$ & $-\sin \alpha / \sin \beta$ & $\cos \alpha / \sin \beta$ \\
$\tilde{u}$ & $H$ & $\sin \alpha / \sin \beta$ & $\cos (\alpha+\beta)$ & $\cos \alpha / \sin \beta$ & $\sin \alpha / \sin \beta$ \\
& $A$ & 0 & 0 & -1 & $1 / \operatorname{tg} \beta$ \\
& $h$ & $-\sin \alpha / \cos \beta$ & $-\sin (\alpha+\beta)$ & $\cos \alpha / \cos \beta$ & $-\sin \alpha / \cos \beta$ \\
$\tilde{d}$ & $H$ & $\cos \alpha / \cos \beta$ & $\cos (\alpha+\beta)$ & $\sin \alpha / \cos \beta$ & $\cos \alpha / \cos \beta$ \\
& $A$ & 0 & 0 & -1 & $\operatorname{tg} \beta$ \\
\hline \hline
\end{tabular}

originating from the SUSY-QCD corrections in detail for representative MSSM scenarios. In Sec. V we conclude.

\section{HIGGS BOSON DECAYS INTO BOTTOM QUARK PAIRS}

\section{A. QCD corrections}

The partial decay widths of the neutral Higgs bosons $\Phi$ $=h, H, A$ into bottom quark pairs, including $\mathrm{QCD}$ corrections, can be cast into the form

$$
\Gamma[\Phi \rightarrow b \bar{b}]=\frac{3 G_{F} M_{\Phi}}{4 \sqrt{2} \pi} \bar{m}_{b}^{2}\left(M_{\Phi}\right)\left(g_{b}^{\Phi}\right)^{2}\left[\Delta_{\mathrm{QCD}}+\Delta_{t}^{\Phi}\right],
$$

where regular quark mass effects are neglected. The large logarithmic part of the QCD corrections has been absorbed in the running bottom quark mass $\bar{m}_{b}\left(M_{\Phi}\right)$ defined in the modified minimal subtraction $(\overline{\mathrm{MS}})$ scheme at the scale of the corresponding Higgs boson mass $M_{\Phi}$. The QCD corrections $\Delta_{\mathrm{QCD}}$ and the top quark induced contributions $\Delta_{t}^{\Phi}$ read as [11]

$$
\begin{aligned}
\Delta_{\mathrm{QCD}}= & +5.67 \frac{\alpha_{s}\left(M_{\Phi}\right)}{\pi}+\left(35.94-1.36 N_{F}\right)\left(\frac{\alpha_{s}\left(M_{\Phi}\right)}{\pi}\right)^{2} \\
& +\left(164.14-25.77 N_{F}+0.259 N_{F}^{2}\right)\left(\frac{\alpha_{s}\left(M_{\Phi}\right)}{\pi}\right)^{3} \\
\Delta_{t}^{h / H}= & \frac{g_{t}^{h / H}}{g_{b}^{h / H}}\left(\frac{\alpha_{s}\left(M_{h / H}\right)}{\pi}\right)^{2} \\
& \times\left[1.57-\frac{2}{3} \log \frac{M_{h / H}^{2}}{M_{t}^{2}}+\frac{1}{9} \log ^{2} \frac{\bar{m}_{b}^{2}\left(M_{h / H}\right)}{M_{h / H}^{2}}\right] \\
\Delta_{t}^{A}= & \frac{g_{t}^{A}}{g_{b}^{A}}\left(\frac{\alpha_{s}\left(M_{A}\right)}{\pi}\right)^{2}\left[3.83-\log \frac{M_{A}^{2}}{M_{t}^{2}}+\frac{1}{6} \log ^{2} \frac{\bar{m}_{b}^{2}\left(M_{A}\right)}{M_{A}^{2}}\right]
\end{aligned}
$$

where $N_{F}=5$ active flavors are taken into account. In the intermediate and large Higgs boson mass regimes the QCD corrections reduce the $b \bar{b}$ decay widths by about $50 \%$ due to the large logarithmic contributions.

\section{B. SUSY-QCD corrections}

In the MSSM the full SUSY-QCD corrections to the fermionic decay modes have been computed at next-to-leading order (NLO) $[9,12]$. In the low-energy limit $M_{\phi}, M_{Z}, m_{b}$ $\ll m_{\tilde{b}_{i}}, m_{\tilde{g}}$ the results can be cast into the simple form 


$$
\begin{aligned}
\Gamma(\phi \rightarrow b \bar{b}) & =\Gamma_{\mathrm{QCD}}(\phi \rightarrow b \bar{b})\left[1+C_{F} C_{\phi} \frac{\alpha_{s}}{\pi}\right] \\
C_{\phi} \rightarrow C_{\phi}^{L E} & =-\kappa_{\phi} m_{\tilde{g}} \mu \operatorname{tg} \beta I\left(m_{\tilde{b}_{1}}^{2}, m_{\widetilde{b_{2}}}^{2}, m_{\tilde{g}}^{2}\right) \\
\kappa_{h} & =1+\frac{1}{\operatorname{tg} \alpha \operatorname{tg} \beta} \\
\kappa_{H} & =1-\frac{\operatorname{tg} \alpha}{\operatorname{tg} \beta} \\
\kappa_{A} & =1+\frac{1}{\operatorname{tg}^{2} \beta} \\
I(a, b, c) & =-\frac{a b \log \frac{a}{b}+b c \log \frac{b}{c}+c a \log \frac{c}{a}}{(a-b)(b-c)(c-a)} .
\end{aligned}
$$

$\Gamma_{\mathrm{QCD}}(\phi \rightarrow b \bar{b})$ denotes the QCD-corrected decay width of Eq. (6). It should be noted that NLO terms involving the trilinear mixing parameter $A_{b}$ are absent in Eq. (8).

\section{EFFECTIVE LAGRANGIAN AND RESUMMATION}

\section{A. Construction of the effective Lagrangian}

The result of Eq. (8) can be derived from the effective low-energy Lagrangian ${ }^{3}$ [8]

$$
\begin{aligned}
\mathcal{L}_{e f f}= & -\lambda_{b} \overline{b_{R}}\left[\phi_{1}^{0}+\frac{\Delta m_{b}}{\operatorname{tg} \beta} \phi_{2}^{0 *}\right] b_{L}+\text { h.c. } \\
= & -m_{b} \bar{b}\left[1+i \gamma_{5} \frac{G^{0}}{v}\right] b-\frac{m_{b} / v}{1+\Delta m_{b}} \bar{b}\left[g_{b}^{h}\left(1-\frac{\Delta m_{b}}{\operatorname{tg} \alpha \operatorname{tg} \beta}\right) h\right. \\
& \left.+g_{b}^{H}\left(1+\Delta m_{b} \frac{\operatorname{tg} \alpha}{\operatorname{tg} \beta}\right) H-g_{b}^{A}\left(1-\frac{\Delta m_{b}}{\operatorname{tg}^{2} \beta}\right) i \gamma_{5} A\right] b
\end{aligned}
$$

with

\footnotetext{
${ }^{3}$ This effective Lagrangian has been obtained by integrating out the heavy SUSY particles $\widetilde{b}, \tilde{g}$ and is thus not restricted to large values of $\operatorname{tg} \beta$ only. It should be noted that the scale dependence of the running bottom mass and Yukawa coupling is purely QCD initiated, since the heavy SUSY particles are integrated out at a fixed scale of $\mathcal{O}\left(M_{S U S Y}\right)$ and thus do not appear as active partons in the corresponding renormalization group equations.
}

$$
\begin{aligned}
\Delta m_{b} & =\frac{C_{F}}{2} \frac{\alpha_{s}}{\pi} m_{\tilde{g}} \mu \operatorname{tg} \beta I\left(m_{\tilde{b}_{1}}^{2}, m_{\tilde{b}_{2}}^{2}, m_{\tilde{g}}^{2}\right) \\
m_{b} & =\frac{\lambda_{b} v_{1}}{\sqrt{2}}\left[1+\Delta m_{b}\right] \\
\phi_{1}^{0} & =\frac{1}{\sqrt{2}}\left[v_{1}+H \cos \alpha-h \sin \alpha+i A \sin \beta-i G^{0} \cos \beta\right] \\
\phi_{2}^{0} & =\frac{1}{\sqrt{2}}\left[v_{2}+H \sin \alpha+h \cos \alpha+i A \cos \beta+i G^{0} \sin \beta\right]
\end{aligned}
$$

after expansion up to NLO. The symbol $\phi_{1}^{0}\left(\phi_{2}^{0}\right)$ denotes the neutral components of the Higgs boson doublet coupling to down-type (up-type) quarks. The parameter $\operatorname{tg} \beta=v_{2} / v_{1}$ is defined as the ratio of the two vacuum expectation values, and $v^{2}=v_{1}^{2}+v_{2}^{2}=1 / \sqrt{2} G_{F}$ is related to the Fermi constant $G_{F}$. The would-be Goldstone field $G^{0}$ is absorbed by the $Z$ boson and generates its longitudinal component. The SUSYQCD corrections turn out to be significant for large values of $\operatorname{tg} \beta$ and moderate or large $\mu$ values. In order to improve the perturbative result all terms of $\mathcal{O}\left[\left(\alpha_{s} \mu \operatorname{tg} \beta\right)^{n}\right]$ have been resummed [8]. The correctly resummed effective Lagrangian is given by Eq. (9). The correction $\Delta m_{b}$ is non-decoupling in the sense that scaling all SUSY parameters $m_{\tilde{b}_{1,2}}, m_{\tilde{g}}, \mu$ in Eq. (10) leaves $\Delta m_{b}$ invariant. However, its contribution develops decoupling properties [13], as we will discuss later on.

Apart from the correction $\Delta m_{b}$ there is a second class of potentially large (non-decoupling) contributions at higher orders which may spoil the perturbative reliability of the results: The trilinear mixing parameter $A_{b}$ can be of similar size as $\mu \operatorname{tg} \beta$ as e.g. in no-mixing scenarios of the sbottom particles. In the low-energy limit of Eq. (8) such terms are absent. However, they arise at higher orders. In the following we develop an approach to include $A_{b}$ terms in the resummation of Eq. (9). For this purpose we start from the unrenormalized effective Lagrangian in the low-energy limit at leading order:

$$
\mathcal{L}_{\text {eff }}^{L O}=-\lambda_{b}^{0} \bar{b}_{R}^{0} \phi_{1}^{0} b_{L}^{0}+\text { h.c. }
$$

Including higher-order corrections in the low-energy limit, the pole mass $m_{b}$ of the bottom quark is given by

$$
m_{b}=\frac{\lambda_{b}^{0}}{\sqrt{2}} v_{1}+\Sigma_{b}\left(m_{b}\right)
$$

where the self-energy $\Sigma_{b}\left(m_{b}\right)$ can be decomposed as

$$
\Sigma_{b}\left(m_{b}\right)=\frac{\lambda_{b}^{0}}{\sqrt{2}}\left[\Delta_{1} v_{1}+\Delta_{2} v_{2}\right]=\frac{\lambda_{b}^{0}}{\sqrt{2}} v_{1}\left[\Delta_{1}+\Delta_{2} \operatorname{tg} \beta\right]
$$

The leading parts in $A_{b}$ and $\mu$ are finite at NLO, 


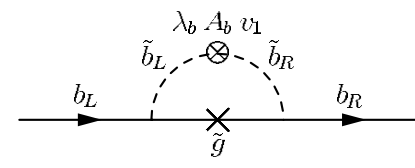

(a)

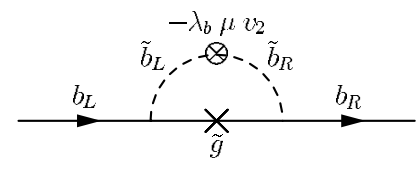

(b)
FIG. 1. One-loop contribution to the quantities (a) $\Delta_{1}$ and (b) $\Delta_{2}$.

$$
\begin{aligned}
& \Delta_{1}=-\frac{C_{F}}{2} \frac{\alpha_{s}}{\pi} m_{\tilde{g}} A_{b} I\left(m_{\tilde{b}_{1}}^{2}, m_{\tilde{b}_{2}}^{2}, m_{\tilde{g}}^{2}\right) \\
& \Delta_{2}=\frac{C_{F}}{2} \frac{\alpha_{s}}{\pi} m_{\tilde{g}} \mu I\left(m_{\tilde{b}_{1}}^{2}, m_{\tilde{b}_{2}}^{2}, m_{\tilde{g}}^{2}\right)=\frac{\Delta m_{b}}{\operatorname{tg} \beta} .
\end{aligned}
$$

Inserting these two expressions in Eq. (12) leads to the wellknown result that the radiative corrections to the bottom mass are proportional to $A_{b}-\mu \operatorname{tg} \beta$, i.e. the off-diagonal components of the sbottom mass matrix of Eq. (2).

The structure of the self-energy beyond NLO can be derived from general arguments based on the asymptotic behavior of the corresponding Feynman diagrams in the lowenergy limit. The terms involving $A_{b}$ or $\mu$ are generated by mass insertions in the virtual sbottom propagators. At NLO the diagrams of Fig. 1 behave asymptotically as ${ }^{4}$

$$
\begin{gathered}
\alpha_{s} \lambda_{b}\left(A_{b} v_{1}-\mu v_{2}\right) m_{\tilde{g}} \times C_{0}\left(0,0 ; m_{\tilde{b}_{1}}, m_{\tilde{b}_{2}}, m_{\tilde{g}}\right) \\
\sim \alpha_{s} m_{b} m_{\tilde{g}} \frac{A_{b}-\mu \operatorname{tg} \beta}{M_{S U S Y}^{2}}
\end{gathered}
$$

(for $M_{S U S Y} \sim m_{\tilde{b}_{1}} \sim m_{\tilde{b}_{2}} \sim m_{\tilde{g}}$ ) coinciding with the explicit results of Eq. (14). At next-to-next-to-leading order (NNLO) the leading contributions involving $A_{b}$ and $\mu$ are generated by e.g. the diagrams of Fig. 2. The diagrams (a) and (b) behave asymptotically as

$$
\begin{gathered}
\alpha_{s}^{2} \lambda_{b}\left(A_{b} v_{1}-\mu v_{2}\right) m_{g} A_{0}\left(m_{\tilde{b}_{i}}\right) D_{0}\left(0,0,0 ; m_{\tilde{b}_{1}}, m_{\widetilde{b}_{2}}, m_{\tilde{b}_{j}}, m_{g}\right) \\
\sim \alpha_{s}^{2} m_{b} m_{\tilde{g}} \frac{A_{b}-\mu \operatorname{tg} \beta}{M_{S U S Y}^{2}}
\end{gathered}
$$

while diagram (c) develops the low-energy behavior

$$
\begin{aligned}
& \alpha_{s}^{2} \lambda_{b}\left(A_{b} v_{1}-\mu v_{2}\right) m_{\tilde{g}} B_{0}\left(0 ; m_{\tilde{b}_{1}}, m_{\tilde{b}_{2}}\right) C_{0}\left(0,0 ; m_{\tilde{b}_{i}}, m_{\tilde{b}_{j}}, m_{\tilde{g}}\right) \\
& \sim \alpha_{s}^{2} m_{b} m_{\tilde{g}} \frac{A_{b}-\mu \operatorname{tg} \beta}{M_{S U S Y}^{2}}
\end{aligned}
$$

Thus, the diagrams of Fig. 2 contribute to the same order as the pure QCD corrections to the NLO results and do not generate leading terms of $\mathcal{O}\left(A_{b}^{2}\right), \quad \mathcal{O}\left(\mu^{2} \operatorname{tg}^{2} \beta\right)$ nor

\footnotetext{
${ }^{4}$ The functions $A_{0}, B_{0}, C_{0}, D_{0}$ denote the usual one-loop scalar integrals for one-, two-, three- and four-point functions.
}

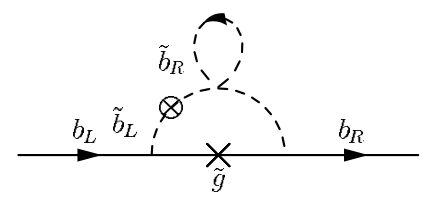

(a)

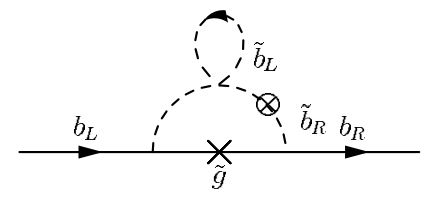

(b)

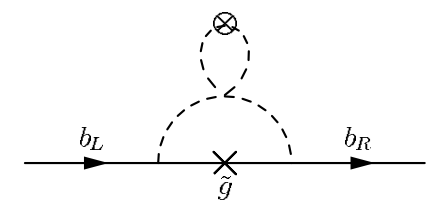

(c)

FIG. 2. Non-decoupling two-loop contributions to $\Delta_{1}$ and $\Delta_{2}$.

$\mathcal{O}\left(A_{b} \mu \operatorname{tg} \beta\right)$. This power-counting argument can be applied to all other two-loop diagrams involving $\mu$ and $A_{b}$, too. Any further mass insertion is suppressed by another power of $m_{b} / M_{S U S Y}$, and is therefore non-leading.

These arguments can be extended to any perturbative order. Due to the Kinoshita-Lee-Nauenberg theorem $[14,15]$ irreducible diagrams do not develop power-like divergences in the bottom mass for $m_{b} \rightarrow 0$. Any mass insertion in the sbottom propagators leads to the replacement

$$
\begin{gathered}
\frac{1}{q^{2}-m_{\tilde{b}_{i}^{2}}} \rightarrow \frac{1}{q^{2}-m_{\tilde{b}_{1}^{2}}} m_{b}\left(A_{b}-\mu \operatorname{tg} \beta\right) \frac{1}{q^{2}-m_{\tilde{b}_{2}^{2}}} \\
\sim-\frac{m_{b}\left(A_{b}-\mu \operatorname{tg} \beta\right)}{M_{S U S Y}^{2}} \frac{1}{q^{2}-m_{\tilde{b}_{i}^{2}}} .
\end{gathered}
$$

Therefore, the low-energy behavior of the mass-inserted diagram is modified by an additional power of $m_{b}\left(A_{b}-\mu \operatorname{tg} \beta\right) / M_{S U S Y}^{2}$. Consequently, the diagrams of Fig. 2 constitute the leading contributions in $A_{b}$ and $\mu \operatorname{tg} \beta$ at NNLO. These arguments prove that the results of Eq. (14) include all leading powers of $\alpha_{s} A_{b}$ and $\alpha_{s} \mu \operatorname{tg} \beta$. This is confirmed by the explicit two-loop results of Ref. [16].

In order to obtain the effective low-energy Lagrangian from the expression (12) for the bottom mass, we have to perform the replacements $v_{1} \rightarrow \sqrt{2} \phi_{1}^{0}$ and $v_{2} \rightarrow \sqrt{2} \phi_{2}^{0 *}$ in the corresponding bottom mass operator. These replacements lead to the exact interactions with non-propagating Higgs fields, i.e. in the low-energy limit of small Higgs boson momentum [17]. The final expression of the effective Lagrangian can be cast into the form

$$
\mathcal{L}_{e f f}=-\lambda_{b}^{0} \bar{b}_{R}^{0}\left\{\left(1+\Delta_{1}\right) \phi_{1}^{0}+\Delta_{2} \phi_{2}^{0 *}\right\} b_{L}^{0}+\text { h.c. }
$$

which differs from previous results by the new factor $\left(1+\Delta_{1}\right)$ in front of $\phi_{1}^{0}$. This expression has to be matched with the renormalized low-energy Lagrangian 


$$
\mathcal{L}_{e f f}=-\lambda_{b} \overline{b_{R}}\left\{\phi_{1}^{0}+\frac{\Delta_{b}}{\operatorname{tg} \beta} \phi_{2}^{0 *}\right\} b_{L}+\text { h.c. }
$$

yielding the relations ${ }^{5}$

$$
\begin{aligned}
& \lambda_{b}=\lambda_{b}^{0}\left(1+\Delta_{1}\right) \\
& \Delta_{b}=\frac{\Delta_{2} \operatorname{tg} \beta}{1+\Delta_{1}}=\frac{\Delta m_{b}}{1+\Delta_{1}} .
\end{aligned}
$$

Thus all terms of $\mathcal{O}\left[\left(\alpha_{s} / M_{S U S Y}\right)^{n}(\mu \operatorname{tg} \beta)^{m} A_{b}^{n-m}\right]$ are resummed by means of the simple replacement

$$
\Delta m_{b} \rightarrow \frac{\Delta m_{b}}{1+\Delta_{1}}
$$

in the effective Lagrangian of Eq. (9). This proof confirms and extends the resummation presented in Ref. [8] and explains the absence of any $A_{b}$ terms in Eq. (8) in terms of a clear physical interpretation: the leading $A_{b}$ terms are absorbed in the definition of the effective Yukawa coupling $\lambda_{b}$ in the low-energy effective Lagrangian. In a Feynman diagrammatic approach this corresponds to a cancellation of the $A_{b}$ terms in the bottom-mass counterterms and the genuine irreducible three-point diagrams. This cancellation is exact at zero-momentum transfer, but a mild dependence on $A_{b}$ appears when keeping all external momenta on-shell due to the momentum dependence of the one-particle-irreducible (1PI) three-point functions.

The final results for the resummed partial decay widths can be cast into the form ${ }^{6}$ [see Eqs. (6)-(8)]

$$
\begin{aligned}
\Gamma[\Phi \rightarrow b \bar{b}]= & \frac{3 G_{F} M_{\Phi}}{4 \sqrt{2} \pi} \bar{m}_{b}^{2}\left(M_{\Phi}\right)\left[\Delta_{\mathrm{QCD}}+\Delta_{t}^{\Phi}\right] \\
& \times \widetilde{g}_{b}^{\Phi}\left[\widetilde{g}_{b}^{\Phi}+g_{b}^{\Phi}\left(C_{\phi}-C_{\phi}^{L E}\right) \frac{\alpha_{s}}{\pi}\right]
\end{aligned}
$$

with the resummed couplings [see Eqs. (9),(19),(20)]

\footnotetext{
${ }^{5}$ It should be noted that the bottom wave-function renormalization constants do not contain any leading non-decoupling contribution in $A_{b}$ and $\mu$. Moreover, it should be emphasized that the combination $A_{b}-\mu \operatorname{tg} \beta$ only appears in the definition of the bottom mass, while $A_{b}$ and $\mu \operatorname{tg} \beta$ contribute in a different way to the bottom Yukawa coupling and Higgs boson decay processes.

${ }^{6}$ In order to avoid an artificial singularity in $\Gamma(h \rightarrow b \bar{b})$ for vanishing $\alpha$ the remainder proportional to $\left(C_{\phi}-C_{\phi}^{L E}\right)$ is multiplied by the unresummed Yukawa coupling $g_{b}^{\phi}$.
}

$$
\begin{aligned}
& \widetilde{g}_{b}^{h}=\frac{g_{b}^{h}}{1+\Delta_{b}}\left(1-\frac{\Delta_{b}}{\operatorname{tg} \alpha \operatorname{tg} \beta}\right) \\
& \widetilde{g}_{b}^{H}=\frac{g_{b}^{H}}{1+\Delta_{b}}\left(1+\Delta_{b} \frac{\operatorname{tg} \alpha}{\operatorname{tg} \beta}\right) \\
& \widetilde{g}_{b}^{A}=\frac{g_{b}^{A}}{1+\Delta_{b}}\left(1-\frac{\Delta_{b}}{\operatorname{tg}^{2} \beta}\right) .
\end{aligned}
$$

\section{B. Validity of the low-energy approximation}

The expression in Eq. (19) resums the terms of $\mathcal{O}\left[\left(\alpha_{S} / M_{S U S Y}\right)^{n}(\mu \operatorname{tg} \beta)^{m} A_{b}^{n-m}\right]$ to all orders in perturbation theory. However, there are other kinds of non-decoupling terms in the 1PI self-energies, as can be inferred already from the NNLO expressions of Eqs. (16),(17). The question about the numerical size of these non-leading terms arises, and whether the NNLO resummation is necessary in practical applications. Equations (15)-(17) imply that the irreducible NNLO corrections $\Delta_{1}^{(2)}$ and $\Delta_{2}^{(2)}$ to the self-energy are of the order of $\Delta_{\{1,2\}}^{(2)} \sim \alpha_{s} \Delta_{\{1,2\}}$, while the reducible diagrams contribute as $\left(\Delta_{\{1,2\}}\right)^{2}$. For the irreducible diagrams to be dominant compared to the reducible ones, the condition $\left(\Delta_{\{1,2\}}\right)^{2} \lesssim\left|\Delta_{\{1,2\}}^{(2)}\right| \sim \alpha_{s}\left|\Delta_{\{1,2\}}\right|$ has to be fulfilled, i.e. $\left|\Delta_{\{1,2\}}\right|$ $\leqslant \alpha_{s} \sim \mathcal{O}(10 \%)$. Therefore, the scenarios with the NNLO 1 PI being dominant lead to $\left|\Delta_{\{1,2\}}^{(2)}\right| \lesssim \mathcal{O}(1 \%)$, so that the NLO corrections are small, and the size of the NNLO corrections is of the same order as the deviation of the full results from the zero-momentum approximation. This argument can be extended to higher orders in perturbation theory. At the $n$-loop level the non-decoupling 1PI diagrams originate from a single vacuum insertion (analogous to the diagrams of Fig. 2) which are of

$$
\mathcal{O}\left(\alpha_{s}^{n} m_{b} m_{g}^{\sim}\left(A_{b}-\mu \operatorname{tg} \beta\right) / M_{S U S Y}^{2}\right) \simeq \alpha_{s}^{n-1} \Delta_{\{1,2\}} .
$$

Hence, they are negligible, because either they are much smaller than the $n$-loop reducible contribution or the numerical value of the leading corrections is small already at NLO.

The trilinear mixing parameter $A_{b}$ cannot be much larger than $M_{S U S Y}$, since otherwise the color and charge symmetries would be broken [18]. Thus, the contribution $\Delta_{1}$ of Eq. (14) reaches maximal values of $\mathcal{O}(10 \%)$, while the term $\Delta m_{b}$ can be larger by an order of magnitude.

In Fig. 3 we compare the relative NLO corrections including the resummation of $\Delta m_{b}$ with the novel NNLO contributions $\Delta_{1}$ of Eq. (10) as a function of the pseudoscalar Higgs boson mass $M_{A}$ for all three neutral Higgs states in the following MSSM scenario with large $A_{b}$ :

$$
\begin{aligned}
\operatorname{tg} \beta & =30 \\
M_{\tilde{Q}} & =2 \mathrm{TeV} \\
M_{\tilde{g}} & =1.6 \mathrm{TeV} \\
A_{t} & =\mu \cot \beta \\
A_{b} & =-\mu \operatorname{tg} \beta \\
\mu & =-150 \mathrm{GeV} .
\end{aligned}
$$



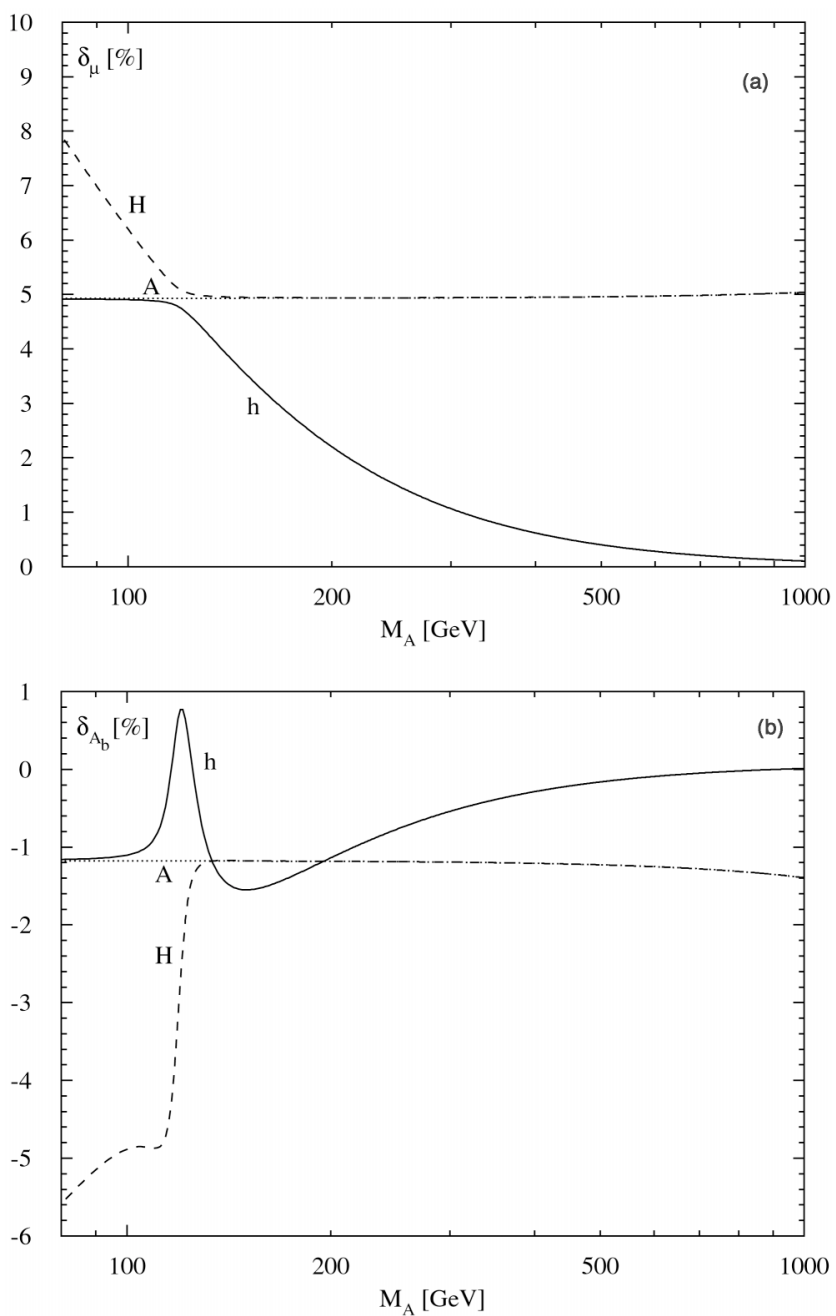

FIG. 3. Relative corrections due to (a) the SUSY-QCD corrections including the resummation of $\Delta m_{b}$ of Eq. (10) and (b) due to $\Delta_{1}$ of Eq. (14) as a function of the pseudoscalar mass $M_{A}$ for all neutral Higgs bosons. The relative corrections are normalized to the QCD-corrected decay widths $\Gamma_{\mathrm{QCD}}(\phi \rightarrow b \bar{b})$ of Eq. (6) in both cases.

The relative corrections are normalized to the QCDcorrected decay widths $\Gamma_{\mathrm{QCD}}(\phi \rightarrow b \bar{b})$ of Eq. (6) in both cases. While the $\Delta m_{b}$ effects are of $\mathcal{O}(10 \%)$ and thus of moderate size, the novel $\Delta_{1}$ contributions turn out to be of $\mathcal{O}(1 \%)$ apart from the small heavy scalar Higgs boson mass range, where they can reach a similar magnitude as the $\Delta m_{b}$ terms. This particular scenario, however, has to be considered as an extreme case. In general the $\Delta_{1}$ terms are small, confirming the previous qualitative discussion.

\section{NUMERICAL RESULTS}

The numerical analysis of the neutral Higgs boson decays into bottom quark pairs is performed for the "small $\alpha_{\text {eff }}$ " MSSM scenario [19] as a representative case:

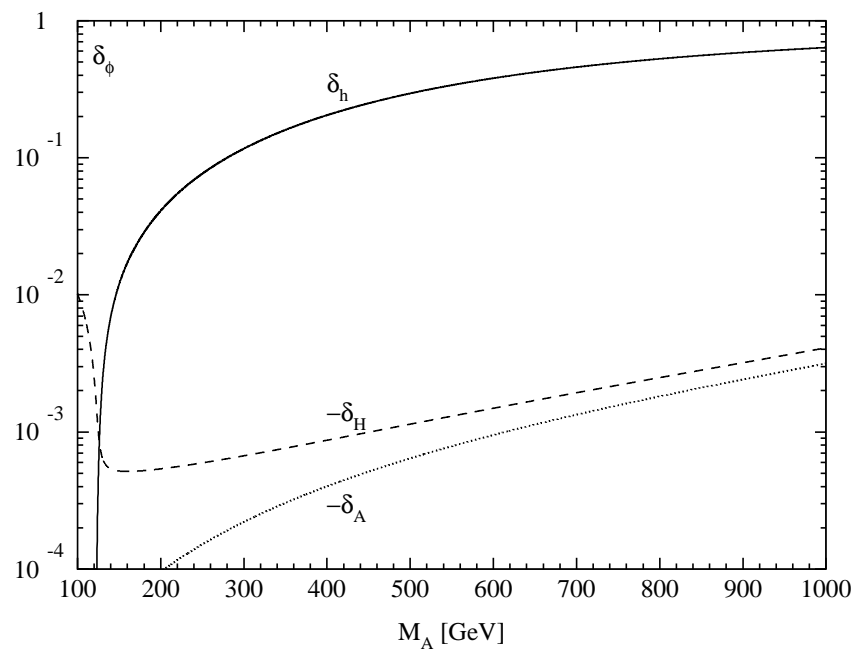

FIG. 4. Relative deviations $\delta_{\phi}$ of the approximate low-energy one-loop result from the full NLO expression as a function of the pseudoscalar mass $M_{A}$ in the "small $\alpha_{e f f}$ " scenario for all neutral Higgs bosons. For the heavy scalar and pseudoscalar Higgs bosons the deviations are negative. The values shown have to be changed in sign.

$$
\begin{aligned}
\operatorname{tg} \beta & =30 \\
M_{\tilde{Q}} & =800 \mathrm{GeV} \\
M_{\tilde{g}} & =500 \mathrm{GeV} \\
M_{2} & =500 \mathrm{GeV} \\
A_{b} & =A_{t}=-1.133 \mathrm{TeV} \\
\mu & =2 \mathrm{TeV} .
\end{aligned}
$$

We use the renormalization-group-improved two-loop expressions of Ref. [20]. The bottom quark pole mass has been chosen to be $M_{b}=4.62 \mathrm{GeV}$, which corresponds to a $\overline{\mathrm{MS}}$ mass $\bar{m}_{b}\left(\bar{m}_{b}\right)=4.28 \mathrm{GeV}$. The strong coupling constant has been normalized to $\alpha_{s}\left(M_{Z}\right)=0.119$.

The resummation effects discussed in the previous section have been derived in the low-energy limit $M_{\phi}^{2}, M_{Z}^{2}, m_{b}^{2}$ $\ll M_{S U S Y}^{2}$. The question arises, how reliable this approximation works in phenomenological applications. In particular, the magnitude of $\mathcal{O}\left(M_{\phi}^{2} / M_{S U S Y}^{2}, M_{Z}^{2} / M_{S U S Y}^{2}, m_{b}^{2} / M_{S U S Y}^{2}\right)$ terms matters for sizeable masses of the low-energy particles. This can be tested explicitly by comparing the approximate results of Eq. (8) with the full one-loop result. A typical example is depicted in Fig. 4 for the "small $\alpha_{e f f}$ " scenario, where the relative difference between the full and approximate one-loop contributions [see Eq. (8)] 


$$
\delta_{\phi}=\frac{C_{\phi}-C_{\phi}^{L E}}{C_{\phi}}
$$

is presented for all neutral Higgs particles as a function of the pseudoscalar Higgs boson mass $M_{A}$. It is clearly visible that the approximation turns out to be sufficient for the heavy neutral Higgs particles $H, A$, but fails for the light scalar Higgs boson $h$ in the decoupling limit [21]. However, in the decoupling limit the size of the approximate SUSY-QCD corrections strongly decreases, since $\operatorname{tg} \alpha \rightarrow-1 / \operatorname{tg} \beta$ and thus

$$
\frac{1}{1+\Delta m_{b}}\left(1-\frac{\Delta m_{b}}{\operatorname{tg} \alpha \operatorname{tg} \beta}\right) \rightarrow 1
$$

so that the SUSY-QCD corrections become negligible. Due to this behavior the low-energy approximation is sufficient for most phenomenological applications. This also explains the failure of the approximation in this case: the large nondecoupling contributions from $\Delta m_{b}$ cancel to a large extent in the lightest Higgs boson couplings, leaving a small remainder of the same order as the non-leading contributions. On the other hand, this cancellation does not occur for the heavy Higgs bosons, and the effective Lagrangian approach yields a good approximation.

There are two basic sources of systematic uncertainties originating from the SUSY-QCD contributions:

(i) The MSSM masses and couplings involved in the NLO SUSY-QCD corrections will only be known with a sizeable uncertainty at the Large Hadron Collider, while future $e^{+} e^{-}$ linear colliders in the $500 \mathrm{GeV}-1 \mathrm{TeV}$ range will enable precision measurements of the SUSY masses and couplings. These errors in the input parameters generate systematic uncertainties for the prediction of the partial decay widths.

(ii) Due to missing higher order results the scale dependence of the strong coupling constant $\alpha_{s}$ will not be compensated. The scale variation yields an estimate of the purely theoretical SUSY-QCD uncertainty, which will be analyzed quantitatively in this section. ${ }^{7}$

The central scale $\mu_{0}$ of the strong coupling constant appearing in the SUSY-QCD corrections will be chosen as the average mass of the involved SUSY particles, i.e.

$$
\mu_{0}=\frac{m_{\tilde{b}_{1}}+m_{\tilde{b}_{2}}+m_{\tilde{g}}}{3}
$$

In order to estimate the residual scale dependence the scale of $\alpha_{s}$ will be varied between $\mu_{0} / 3$ and $3 \mu_{0}$. The usual QCD corrections have been included up to the three-loop order so that the residual purely QCD-induced scale dependence ranges below the per-mille level and can thus safely be neglected.

\footnotetext{
${ }^{7}$ The electroweak contributions introduce additional uncertainties, which are not taken into account. They provide contributions to $\Delta_{1}$ and $\Delta_{2}$ in addition to the SUSY-QCD part.
}
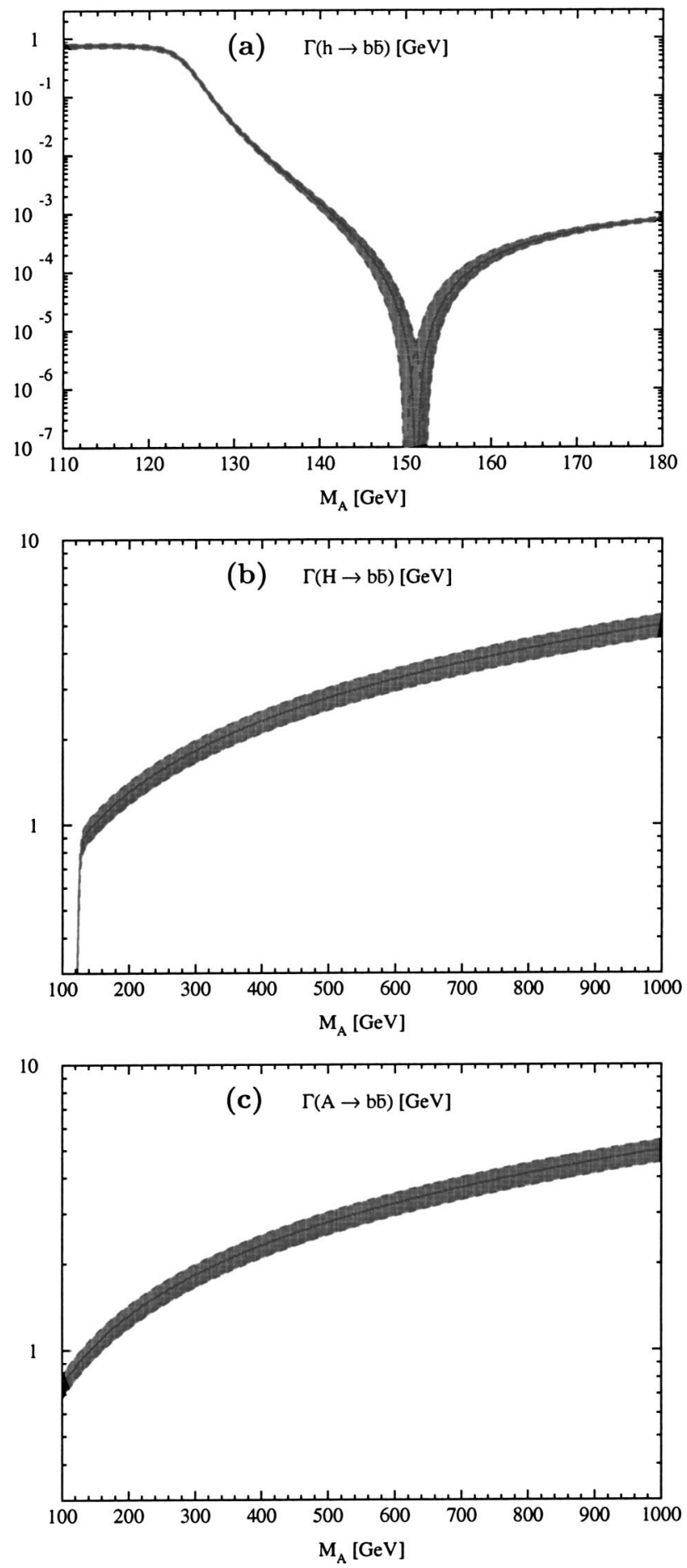

FIG. 5. Partial decay widths $\Gamma(\phi \rightarrow b \bar{b})$ of (a) the light scalar, (b) the heavy scalar and (c) the pseudoscalar Higgs boson in the "small $\alpha_{\text {eff }}$ " scenario. The shaded bands reflect the uncertainties due to the scale choice of the strong coupling constant $\alpha_{s}$.

The results for the partial decay widths are shown in Fig. 5a for the light scalar Higgs boson, in Fig. 5b for the heavy scalar Higgs boson and in Fig. 5c for the pseudoscalar Higgs boson. These results include the QCD corrections up to nextto-next-to-next-to-leading order NNNLO of Eq. (6) and the full NLO SUSY-QCD corrections of Eq. (8) with the resum- 

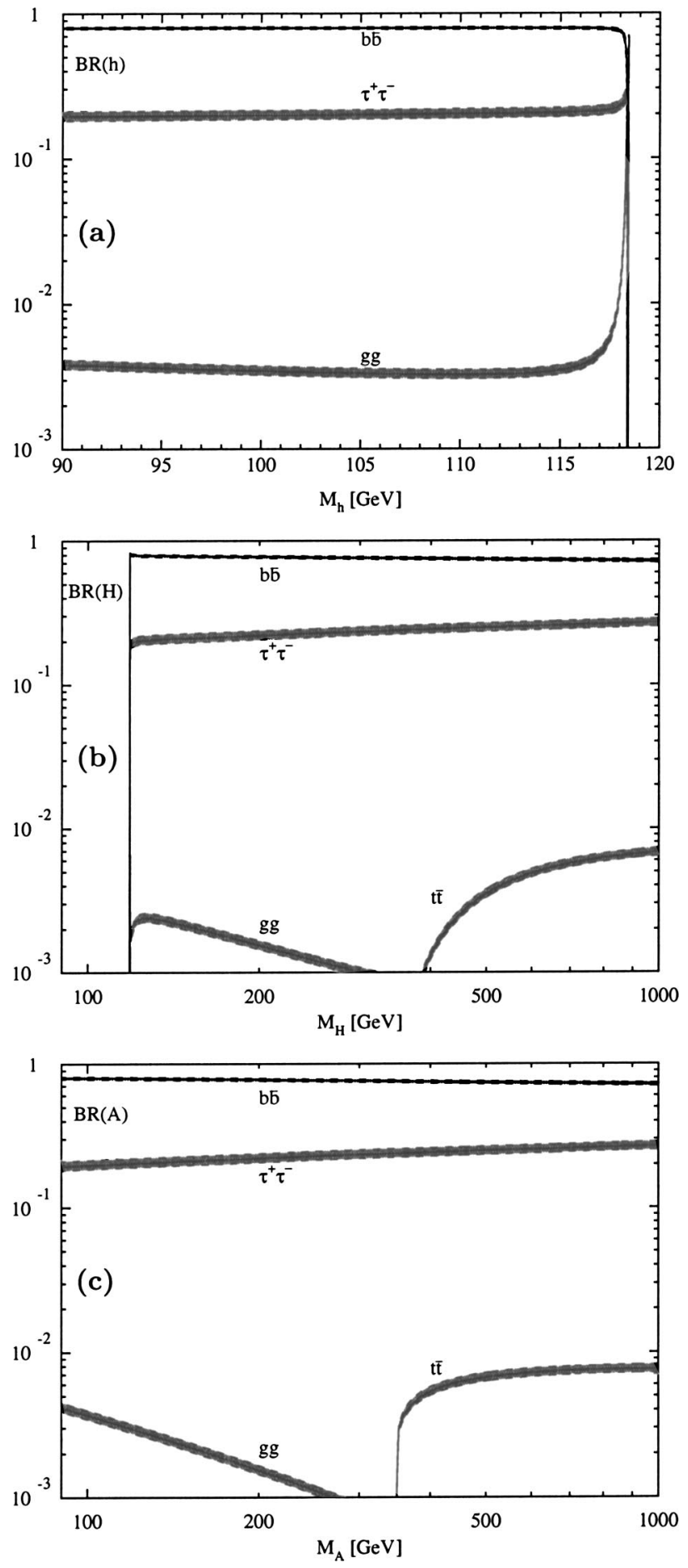

FIG. 6. Branching ratios of (a) the light scalar, (b) the heavy scalar and (c) the pseudoscalar Higgs boson in the "small $\alpha_{e f f}$ " scenario. The shaded bands reflect the uncertainties due to the scale choice of the strong coupling constant $\alpha_{s}$.

mation of the leading $\Delta m_{b}$ and $\Delta_{1}$ terms according to Eqs. (9),(21). It can clearly be inferred from these figures that the remaining uncertainties due to the scale choice are typically of the order of $10 \%$. However, they are significantly enhanced in regions where the SUSY-QCD corrections become large, as in the "small $\alpha_{e f f}$ " scenario, which develops a strongly suppressed partial decay width $\Gamma(h \rightarrow b \bar{b})$ for pseu- doscalar masses $M_{A} \sim 150 \mathrm{GeV}{ }^{8}$ This, however, corresponds only to a tiny region in the light scalar Higgs boson mass $M_{h}$ close to its upper limit for large $M_{A}$ within the "small $\alpha_{e f f}$ " scenario. The theoretical uncertainties turn out to be large at $M_{A} \sim 150 \mathrm{GeV}$.

The uncertainties in the partial decay widths $\Gamma(\phi \rightarrow b \bar{b})$ translate into systematic errors in the corresponding branching ratios. They are depicted in Figs. $6 a-6 c$ for the three neutral Higgs bosons. These results have been obtained with the program HDECAY [22] after including the results obtained in this analysis. Since the partial decay into $b \bar{b}$ pairs is dominant in nearly the entire Higgs boson mass ranges, its uncertainty due to the scale choice above reduces to a level of $\mathcal{O}(1 \%)$. However, the scale dependence of $\Gamma(\phi \rightarrow b \bar{b})$ develops significant systematic errors in the non-leading branching ratios into $\tau^{+} \tau^{-}$, gluon and $t \bar{t}$ pairs. These can reach a level of $\mathcal{O}(10 \%)$ and are larger than the expected experimental accuracy at future $e^{+} e^{-}$linear colliders, which clearly calls for a NNLO calculation of the SUSY-QCD part. These theoretical errors have to be added to the uncertainties due to inaccuracies of the input parameters as presented in [23] and the theoretical errors of the Higgs boson masses and couplings [3]. ${ }^{9}$ They constitute a significant source of uncertainty. An analogous analysis is required for the theoretical uncertainties due to the SUSY-electroweak corrections beyond NLO. However, this is beyond the scope of our paper.

\section{CONCLUSIONS}

In this paper we have reanalyzed the neutral scalar Higgs boson decays into $b \bar{b}$ pairs in the MSSM with particular emphasis on the SUSY-QCD corrections and their theoretical uncertainties. We have extended the resummation of large non-decoupling SUSY-QCD corrections of $\mathcal{O}\left(\alpha_{s} \mu \operatorname{tg} \beta / M_{S U S Y}\right)$ by the inclusion of non-decoupling terms of $\mathcal{O}\left(\alpha_{s} A_{b} / M_{S U S Y}\right)$ which have not been taken into account in previous analyses. We have shown that these terms are absent at NLO in the effective Lagrangian but arise at NNLO and beyond. This can easily be traced back to the renormalization of the bottom Yukawa coupling in the lowenergy limit, where the heavy SUSY particles are integrated out. We have obtained the important result that these novel contributions hardly affect the theoretical predictions for the partial decay widths into $b \bar{b}$ pairs so that they do not endanger the reliability of the perturbative result in contrast to the leading terms of $\mathcal{O}\left(\alpha_{s} \mu \operatorname{tg} \beta / M_{S U S Y}\right)$.

We investigated the remaining theoretical uncertainties generated by the SUSY-QCD corrections quantitatively. While the theoretical errors of the partial decay widths

\footnotetext{
${ }^{8}$ The explicit value of the pseudoscalar mass where the Yukawa coupling vanishes depends strongly on the included higher-order corrections.

${ }^{9}$ The uncertainties due to the Higgs boson masses will be eliminated to a large extent, once they will be measured directly in future experiments.
} 
$\Gamma(\phi \rightarrow b \bar{b})$ turn out to be of $\mathcal{O}(10 \%)$, this effect cancels to a large extent in the branching ratios $\mathrm{BR}(\phi \rightarrow b \bar{b})$ due to its dominance. It appears, however, as a sizeable increase in the systematic uncertainties of the non-leading branching ratios into $\tau^{+} \tau^{-}$, gluon and $t \bar{t}$ pairs, which appear to be larger than the anticipated experimental accuracies at future linear $e^{+} e^{-}$ colliders. This clearly calls for a NNLO calculation of the SUSY-QCD part, which is beyond the scope of this work.

\section{ACKNOWLEDGMENTS}

J.G. would like to thank the Max-Planck-Institute für Physik in Munich for their kind hospitality. We are indebted to G. Weiglein for useful discussions. We are grateful to P. Zerwas for carefully reading the manuscript. This work has been supported in part by the Swiss Bundesamt für Bildung und Wissenschaft and by the European Union under contract HPRN-CT-2000-00149.
[1] M. Carena and H.E. Haber, Prog. Part. Nucl. Phys. 50, 63 (2003), and references therein.

[2] LEP Higgs Working Group, hep-ex/0107029; hep-ex/0107030.

[3] S. Heinemeyer, W. Hollik, and G. Weiglein, J. High Energy Phys. 06, 009 (2000).

[4] H. Haber and G. Kane, Phys. Rep. 117, 75 (1985).

[5] A. Djouadi, J. Kalinowski, P. Ohmann, and P.M. Zerwas, Z. Phys. C 74, 93 (1997).

[6] L. Hall, R. Rattazzi, and U. Sarid, Phys. Rev. D 50, 7048 (1994); R. Hempfling, ibid. 49, 6168 (1994); M. Carena, M. Olechowski, S. Pokorski, and C.E.M. Wagner, Nucl. Phys. B426, 269 (1994); D. Pierce, J. Bagger, K. Matchev, and R. Zhang, ibid. B491, 3 (1997).

[7] K.S. Babu and C.F. Kolda, Phys. Lett. B 451, 77 (1999); M. Carena, S. Mrenna, and C.E.M. Wagner, Phys. Rev. D 60, 075010 (1999); J. Guasch, W. Hollik, and S. Peñaranda, Phys. Lett. B 515, 367 (2001); M. Carena, H.E. Haber, H.E. Logan, and S. Mrenna, Phys. Rev. D 65, 055005 (2002); 65, 099902(E) (2002); A. Belyaev, D. Garcia, J. Guasch, and J. Sola, J. High Energy Phys. 06, 059 (2002).

[8] M. Carena, D. Garcia, U. Nierste, and C.E.M. Wagner, Nucl. Phys. B577, 88 (2000).

[9] A. Dabelstein, Nucl. Phys. B456, 25 (1995).

[10] S. Heinemeyer, W. Hollik, and G. Weiglein, Eur. Phys. J. C 16, 139 (2000).

[11] E. Braaten and J.P. Leveille, Phys. Rev. D 22, 715 (1980); N. Sakai, ibid. 22, 2220 (1980); T. Inami and T. Kubota, Nucl. Phys. B179, 171 (1981); S.G. Gorishny, A.L. Kataev, and S.A. Larin, Yad. Fiz. 40, 517 (1984) [Sov. J. Nucl. Phys. 40, 329 (1984)]; M. Drees and K. Hikasa, Phys. Rev. D 41, 1547 (1990); Phys. Lett. B 240, 455 (1990); 262, 497(E) (1991); S.G. Gorishny, A.L. Kataev, S.A. Larin, and L.R. Surguladze, Mod. Phys. Lett. A 5, 2703 (1990); Phys. Rev. D 43, 1633 (1991); A.L. Kataev and V.T. Kim, Mod. Phys. Lett. A 9, 1309
(1994); L.R. Surguladze, Phys. Lett. 341, 61 (1994); K.G. Chetyrkin, J.H. Kühn, and A. Kwiatkowski, Proceedings of the Workshop "QCD at LEP," Aachen, 1994, hep-ph/9407271; K.G. Chetyrkin, Phys. Lett. B 390, 309 (1997); K.G. Chetyrkin and A. Kwiatkowski, Nucl. Phys. B461, 3 (1996); S.A. Larin, T. van Ritbergen, and J.A.M. Vermaseren, Phys. Lett. B 362, 134 (1995).

[12] J.A. Coarasa, R.A. Jimenez, and J. Solà, Phys. Lett. B 389, 312 (1996); H. Eberl, K. Hidaka, S. Kraml, W. Majerotto, and Y. Yamada, Phys. Rev. D 62, 055006 (2000).

[13] H.E. Haber, M.J. Herrero, H.E. Logan, S. Peñaranda, S. Rigolin, and D. Temes, Phys. Rev. D 63, 055004 (2001).

[14] T. Kinoshita, J. Math. Phys. 3, 650 (1962).

[15] T.D. Lee and M. Nauenberg, Phys. Rev. 133, B1549 (1964).

[16] A. Bednyakov, A. Onishchenko, V. Velizhanin, and O. Veretin, Eur. Phys. J. C 29, 87 (2003).

[17] J. Ellis, M.K. Gaillard, and D.V. Nanopoulos, Nucl. Phys. B106, 292 (1976); A.I. Vainshtein, M.B. Voloshin, V.I. Zakharov, and M.A. Shifman, Yad. Fiz. 30, 1368 (1979) [Sov. J. Nucl. Phys. 30, 711 (1979)]; M. Spira, A. Djouadi, D. Graudenz, and P.M. Zerwas, Nucl. Phys. B453, 17 (1995); B.A. Kniehl and M. Spira, Z. Phys. C 69, 77 (1995); W. Kilian, ibid. 69, 89 (1995).

[18] J.M. Frére, D.R.T. Jones, and S. Raby, Nucl. Phys. B222, 11 (1983).

[19] M. Carena, S. Heinemeyer, C.E.M. Wagner, and G. Weiglein, Eur. Phys. J. C 26, 601 (2003).

[20] M. Carena, H.E. Haber, S. Heinemeyer, W. Hollik, C.E. Wagner, and G. Weiglein, Nucl. Phys. B580, 29 (2000).

[21] P. Häfliger, Diploma thesis, ETH Zürich, 2002.

[22] A. Djouadi, J. Kalinowski, and M. Spira, Comput. Phys. Commun. 108, 56 (1998); M. Spira, Fortschr. Phys. 46, 203 (1998).

[23] A. Djouadi, M. Spira, and P.M. Zerwas, Z. Phys. C 70, 427 (1996). 